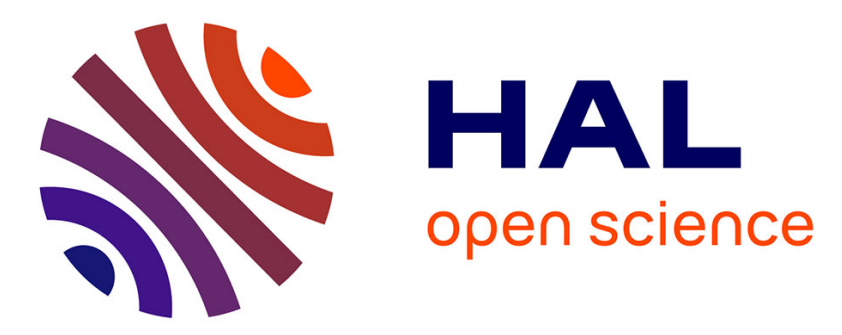

\title{
A condition-based imperfect replacement policy for a periodically inspected system with two dependent wear indicators
}

\author{
Sophie Mercier, Ha Pham
}

\section{- To cite this version:}

Sophie Mercier, Ha Pham. A condition-based imperfect replacement policy for a periodically inspected system with two dependent wear indicators. Applied Stochastic Models in Business and Industry, 2014, 10.1002/asmb.2011 . hal-00984779

\section{HAL Id: hal-00984779 \\ https://hal.science/hal-00984779}

Submitted on 20 Dec 2014

HAL is a multi-disciplinary open access archive for the deposit and dissemination of scientific research documents, whether they are published or not. The documents may come from teaching and research institutions in France or abroad, or from public or private research centers.
L'archive ouverte pluridisciplinaire HAL, est destinée au dépôt et à la diffusion de documents scientifiques de niveau recherche, publiés ou non, émanant des établissements d'enseignement et de recherche français ou étrangers, des laboratoires publics ou privés. 


\title{
A condition-based imperfect replacement policy for a periodically inspected system with two dependent wear indicators
}

\author{
Sophie MERCIER, Hai Ha PHAM \\ Université de Pau et des Pays de l'Adour, \\ Laboratoire de Mathématiques et de leurs Applications - Pau (UMR CNRS 5142), \\ Bâtiment IPRA, Avenue de l'Université, F-64013 PAU cedex, FRANCE \\ sophie.mercier@univ-pau.fr
}

November 26, 2013

\begin{abstract}
A two-component system is considered, which is subject to accumulative deterioration. Due to common stress, the components are dependent. Their joint deterioration is modelled with a bivariate non decreasing Lévy process. The deterioration level of both components is known only through perfect and periodic inspections. By an inspection, components with deterioration level beyond a specific threshold are instantaneously replaced by new ones (corrective or preventive replacements). Otherwise, they are left as they are. Between inspections, failures remain unrevealed. This replacement policy is classical in a univariate setting, with deterioration modelled by a Gamma process. In the bivariate case, it leads to imperfect repairs at the system level, which highly complicates the study. The replacement policy is assessed through cost functions on both finite and infinite horizons, which take into account some economical dependence between components. Markov renewal theory is used to study the behaviour of the system, in a continuous and bivariate setting. Numerical experiments illustrate the study, considering a specific Lévy process with univariate Gamma processes as margins. Though technical details are not provided here for the numerical computations, the paper shows that there is a technical gap between the traditional one-dimensional studies and the present two-dimensional one, especially for the computation of the asymptotic distribution of the underlying Markov chain. Hence there is a need for further development in the bivariate (or multivariate) setting. Keywords: Reliability; multivariate Lévy processes; dependent wear indicators; Gamma processes; Markov renewal theory.
\end{abstract}

\section{Introduction}

In reliability, stochastic models for deterioration based on actual measurements of the system deterioration level have been the subject of many studies during

${ }^{*}$ Corresponding author, Phone: 335594075 37, Fax: 33559407555 
the last decades. In case of non decreasing deterioration, classical models include compound Poisson processes and Gamma processes, according to whether the deterioration is due to isolated shocks or continuous wear accumulation, see [1], [2] or [3] e.g.. These classical models are both univariate non decreasing Lévy processes, also called subordinators. We here consider a two-component system, where the deterioration of each component is measured by a univariate subordinator. Because of a common stressing environment, the deterioration levels of the two components are correlated. Hence the need for a bivariate stochastic model to describe the system evolution.

Up to our knowledge, multivariate non decreasing wear indicators have not been much studied in the previous literature. Several notable exceptions may however be found such as [4] and [5], which both use specific constructions leading to some specific bivariate increasing Lévy processes (though not recognized as such in the quoted papers). Following [6], we here propose to model the evolution of our two-component system by a general bivariate subordinator (or non decreasing bivariate Lévy process). This englobes lots of possible dependence between the two marginal processes. As an example, let us consider two components in a common stressing environment, where the stress arrive by shocks according to a Poisson process. Without considering the common environment, the deterioration of the two components is measured by two univariate and independent subordinators (Gamma processes e.g.). Assume that the common shocks make both components older, with identically and independently distributed bivariate increments of age at each shock. The bivariate "virtual" age of the two components submitted to the shocks then appears as a bivariate compound Poisson process. The process describing the two components submitted to the shocks hence appears as a bivariate Lévy process (composed of the two initial independent Gamma processes) subordinated by a bivariate compound Poisson process, and it consequently is a bivariate subordinator.

Both series and parallel structures are envisioned for the two-component system. Each component is considered as failed as soon at its deterioration level has reached a pre-determined failure threshold. In [6], the system was assumed to be continuously monitored and repairs to be perfect. In the present paper, the deterioration level of the two components is known only through periodic inspections. By an inspection, failed components are instantaneously replaced by new ones (corrective replacements). In case where one single component is down by an inspection, this leads to an imperfect repair at the system level. For the two envisioned structures (series and parallel), the system may remain failed for a while before an inspection. To lower the system down-time, a condition-based maintenance policy is considered, where preventive replacements are performed at inspection times, when the deterioration level of each component is observed to be beyond a preventive threshold (lower than the corrective threshold). The preventive maintenance policy is assessed through a cost function, both on a finite and infinite horizon. This cost function takes into account down-time unitary costs, inspection costs as well as replacement costs, with economical dependence between replacement costs. (Simultaneous replacements are less costly than separate replacements). Our model hence takes into account two kinds of dependence: 1. stochastic dependence between the random deterioration levels of each component (induced by common stress); 2. economical dependence, which may lead to grouped replacements to lower replacement costs, and consequently implies some kind of functional dependence. This twofold dependence 
highly complicates the study (and especially the stochastic one), as well as the imperfect repairs.

Similar preventive and corrective threshold-based replacement policies have already been considered in the literature on a large scale in the univariate setting, see [3] for numerous references in case the system deterioration is modelled by a Gamma process. Papers are much fewer in the multivariate setting. One may however quote [7], where the authors envision a two-unit series system with stochastically independent but economically dependent components, in a discrete time setting. Though their study is highly simplified by the assumption of stochastic independence between components, their condition-based inspection scheme is however more complicated than our periodic one. Also, like lots of other papers on similar subjects, they only envision long-time runs whereas we also consider the case of a finite horizon time, additionally.

The article is organized as follows: the model is presented in Section 2, both for the unmaintained and preventively maintained system. Section 3 is devoted to theoretical developments, based on Markov renewal theory. Numerical experiments illustrate the study in Section 4, considering a specific Lévy process with univariate Gamma processes as margins. Concluding remarks end the paper in Section 5 .

\section{The model}

\subsection{The unmaintained system}

The deterioration of the two-component system is measured by a bivariate nondecreasing Lévy process $X=\left(X_{t}^{(1)}, X_{t}^{(2)}\right)_{t \geq 0}$, also called bivariate subordinator. This means that the process starts from $(0,0)$ and has homogeneous and independent increments, see [8] for more details. As in [6], the process $X$ is assumed to have null drift, so that $X$ is a pure jump process.

For each $i=1,2$, the marginal process $\left(X_{t}^{(i)}\right)_{t \geq 0}$ stands for the deterioration of the $i$-th component and is a univariate subordinator. The $i$-th component is considered as failed as soon as its deterioration level is beyond threshold $L_{i}$ and we set

$$
\sigma^{(i)}=\inf \left(t>0: X_{t}^{(i)}>L_{i}\right)
$$

to be the time-to-failure of the $i$-th component. In the series case, the system time-to-failure is

$$
\sigma_{S}=\min \left(\sigma^{(1)}, \sigma^{(2)}\right) .
$$

In the parallel case, it is

$$
\sigma_{P}=\max \left(\sigma^{(1)}, \sigma^{(2)}\right) .
$$

The respective distributions of $X_{t}$ and $X_{t}^{(i)}$ are denoted by $\mu_{t}$ and $\mu_{t}^{(i)}$, their cumulative distribution functions (c.d.f.) by $F_{t}$ and $F_{t}^{(i)}$, and their survival functions by $\bar{F}_{t}$ and $\bar{F}_{t}^{(i)}$. Note that we do not assume $\mu_{t}$ and $\mu_{t}^{(i)}$ to admit a density with respect to Lebesgue measure.

The state of the system is perfectly controlled via periodic inspections at time $0, T, 2 T, \ldots$ To avoid the trivial case where the system never fails, we assume 
in all the paper that $\mathbb{P}\left(X_{T}^{(1)}>L_{1}, X_{T}^{(2)}>L_{2}\right)=\mathbb{P}\left(\sigma^{(1)} \leq T, \sigma^{(2)} \leq T\right)>0$. Between inspections, failures remain unrevealed. At time $n T, n \geq 0$, only failed components are replaced (corrective replacements). This means that, by an inspection, deterioration levels of failed components are reset to zero whereas they are left unchanged otherwise. Replacements are assumed to be instantaneous and perfect.

\subsection{The preventive maintenance policy}

In order to avoid failures and to shorten down periods, preventive maintenance thresholds $M_{i}$ are next introduced (with $0 \leq M_{i} \leq L_{i}, i=1,2$ ), with a similar replacement policy as for corrective replacements otherwise. More specifically, at time $n T, n \geq 0$, if the deterioration level of the $i^{t h}$ component is between $M_{i}$ and $L_{i}$, a preventive replacement is performed. If its deterioration is beyond $L_{i}$, the component is failed and a corrective replacement takes place. Preventive replacements $(\mathrm{PR})$ are assumed to be instantaneous and perfect, just as for corrective replacements $(\mathrm{CR})$.

This preventive maintenance (PM) policy is illustrated in Figure 1, where there are: two simultaneous corrective replacements at time $T$, one single preventive replacement at times $2 T$ and $3 T$, two simultaneous preventive replacements at times $4 T$ and $5 T$. In this sequence, the maintenance actions at times $2 T$ and $3 T$ are imperfect, at the system level.

Note that though the system state (up or down) depends on its structure (series or parallel), the replacement policy is the same for both structures.

Taking $M_{i}=L_{i}$ for $i=1,2$, the unmaintained system appears as a special case of the preventively maintained system. Taking $M_{i}=0$ for $i=1,2$, the system is replaced every $T$ time units and the classical periodic replacement policy with no repair at failure and period $T$ also is a special case of the PM policy.

To assess the PM policy, cost functions are considered, which takes into account a down-time unitary cost per unit time $\left(c_{u}\right)$, inspection costs $\left(c_{p}\right)$ and replacement costs. The cost of simultaneous replacements of both components is $c_{1}+c_{2}+c_{r}$. If only the $i$-th component is replaced $(i=1,2)$, the cost is $c_{i}+c_{r}$. This induces an economical dependence between cost replacements. Note that we do not consider different costs for preventive or corrective replacements. Indeed, whatever their nature is, all replacements are instantaneously performed at inspection times and there is no special delay for the corrective ones, nor special action either (just replace too degraded components). However, if necessary, the results might easily be adapted in case of different replacement costs.

\section{Theoretical results}

\subsection{Structure of the underlying stochastic process}

Let $Y=\left(Y_{t}^{(1)}, Y_{t}^{(2)}\right)_{t>0}$ be the stochastic process describing the maintained system. Considering the system state after each inspection, the sequence $\left(Y_{n T}\right)_{n \geq 0}$ is a Markov chain with continuous state space $\left[0, M_{1}\right] \times\left[0, M_{2}\right]$. Indeed, regardless of whether the components are replaced or not at inspection time $n T$, their 
future evolution after time $n T$ only depends on their state at time $n T$. This means that $\left(Y_{t}\right)_{t \geq 0}$ is a semi-regenerative process, with $\left(Y_{n T}\right)_{n \geq 0}$ as embedded Markov chain. Both processes $\left(Y_{n T}^{(i)}\right), i=1,2$ have similar properties and also are semi-regenerative processes, with $\left(Y_{n T}^{(i)}\right)_{n \geq 0}, i=1,2$ as embedded Markov chains and state spaces $\left[0, M_{i}\right], i=1,2$.

We next provide the transition kernels of the different Markov chains, denoted by $\mathcal{Q}(x, d y)$ and $Q^{(i)}\left(x_{i}, d y_{i}\right), i=1,2$. Setting $\mathbb{P}_{x}$ (resp. $\left.\mathbb{P}_{x_{i}}\right)$ to refer to the conditioning by $Y_{0}=x\left(\right.$ resp. $\left.Y_{0}^{(i)}=x_{i}\right)$ and $d y=\left(d y_{1}, d y_{2}\right)$, we recall that

$$
\mathcal{Q}(x, d y)=\mathbb{P}\left(Y_{T} \in d y \mid Y_{0}=x\right)=\mathbb{P}_{x}\left(Y_{T} \in d y\right)
$$

for all $x=\left(x_{1}, x_{2}\right) \in\left[0, M_{1}\right] \times\left[0, M_{2}\right]$ and

$$
Q^{(i)}\left(x_{i}, d y_{i}\right)=\mathbb{P}\left(Y_{T}^{(i)} \in d y_{i} \mid Y_{0}^{(i)}=x_{i}\right)=\mathbb{P}_{x_{i}}\left(Y_{T}^{(i)} \in d y_{i}\right)
$$

for all $x_{i} \in\left[0, M_{i}\right], i=1,2$.

In case $x=(0,0)$ or $x_{i}=0$, we just write $\mathbb{P}=\mathbb{P}_{(0,0)}$ or $\mathbb{P}=\mathbb{P}_{0}$ in the following.

Proposition 1 For $i=1,2$, the transition kernel of the Markov chain $\left(Y_{n T}^{(i)}\right)_{n \in \mathbb{N}}$ is given by

$$
Q^{(i)}\left(x_{i}, d y_{i}\right)=\bar{F}_{T}^{(i)}\left(M_{i}-x_{i}\right) \delta_{0}\left(d y_{i}\right)+1_{\left[x_{i}, M_{i}\right]}\left(y_{i}\right) \mu_{T}^{(i)}\left(d y_{i}-x_{i}\right)
$$

for all $x_{i} \in\left[0, M_{i}\right]$, where $\delta_{0}\left(d y_{i}\right)$ stands for the Dirac mass at 0 .

The transition kernel of the Markov chain $\left(Y_{n T}\right)_{n \in \mathbb{N}}$ is given by

$$
\mathcal{Q}(x, d y)=\sum_{i=1}^{4} \mathcal{Q}_{i}(x, d y)
$$

with

$$
\begin{aligned}
& \mathcal{Q}_{1}(x, d y)=\mathbf{1}_{\left[x_{1}, M_{1}\right]}\left(y_{1}\right) \mathbf{1}_{\left[x_{2}, M_{2}\right]}\left(y_{2}\right) \times \mu_{T}\left(d y_{1}-x_{1}, d y_{2}-x_{2}\right), \\
& \mathcal{Q}_{2}(x, d y)=\mathbf{1}_{\left[x_{2}, M_{2}\right]}\left(y_{2}\right) \times\left(\int_{\left(M_{1},+\infty[\right.} \mu_{T}\left(d u_{1}-x_{1}, d y_{2}-x_{2}\right)\right) \delta_{0}\left(d y_{1}\right), \\
& \mathcal{Q}_{3}(x, d y)=\mathbf{1}_{\left[x_{1}, M_{1}\right]}\left(y_{1}\right) \times\left(\int_{\left(M_{2},+\infty[\right.} \mu_{T}\left(d y_{1}-x_{1}, d u_{2}-x_{2}\right)\right) \delta_{0}\left(d y_{2}\right), \\
& \mathcal{Q}_{4}(x, d y)=\bar{F}_{T}\left(M_{1}-x_{1}, M_{2}-x_{2}\right) \times \delta_{0}\left(d y_{1}\right) \delta_{0}\left(d y_{2}\right)
\end{aligned}
$$

for all $x=\left(x_{1}, x_{2}\right) \in\left[0, M_{1}\right] \times\left[0, M_{2}\right]$.

Proof. For $i=1,2$, there are two possible scenarios for the $i^{\text {th }}$ component at time $T$ : either the component is replaced by a new one and its level deterioration is reset to 0 , or it is left as it is. Starting from $x_{i}$, the first scenario happens with the probability

$$
\mathbb{P}_{x_{i}}\left(X_{T}^{(i)}>M_{i}\right)=\bar{F}_{T}^{(i)}\left(M_{i}-x_{i}\right) .
$$


As for the second scenario, it means that the level of the $i^{\text {th }}$ component at time $T$ is $x_{i}+X_{T}^{(i)}$, with $x_{i}+X_{T}^{(i)} \leq M_{i}$. This provides expression Eq.(1) for $Q^{(i)}\left(x_{i}, d y_{i}\right)$.

As for the whole system, at time $T$, there are three possibilities: either no replacement, or one single replacement, or two simultaneous replacements. According to which component is replaced in case of one single replacement (component 1 or 2 ), this leads to four different possible scenarios. As for the second scenario (replacement of component 1 only), we have:

$$
\mathbb{E}_{x}\left[\varphi\left(Y_{T}\right) \mathbf{1}_{\left\{Y_{T}^{(1)}=0 ; Y_{T}^{(2)}=X_{T}^{(2)}\right\}}\right]=\iint_{\mathbb{R}^{2}} \varphi\left(y_{1}, y_{2}\right) \mathcal{Q}_{2}(x, d y)
$$

for all measurable and non negative function $\varphi$ (and all $x$ ), with

$$
\begin{aligned}
& \mathbb{E}_{x}\left[\varphi\left(Y_{T}\right) \mathbf{1}_{\left\{Y_{T}^{(1)}=0 ; Y_{T}^{(2)}=X_{T}^{(2)}\right\}}\right] \\
& =\mathbb{E}_{x}\left[\varphi\left(0, X_{T}^{(2)}\right) \mathbf{1}_{\left\{X_{T}^{(1)}>M_{1} ; X_{T}^{(2)} \leq M_{2}\right\}}\right] \\
& =\iint_{\mathbb{R}_{+}^{2}} \varphi\left(0, x_{2}+z_{2}\right) \mathbf{1}_{\left\{x_{1}+z_{1}>M_{1} ; x_{2}+z_{2} \leq M_{2}\right\}} \mu_{T}\left(d z_{1}, d z_{2}\right) \\
& =\iint_{\mathbb{R}^{2}} \varphi\left(0, y_{2}\right) \mathbf{1}_{\left(M_{1},+\infty[\right.}\left(u_{1}\right) \mathbf{1}_{\left[x_{2}, M_{2}\right]}\left(y_{2}\right) \mu_{T}\left(d u_{1}-x_{1}, d y_{2}-x_{2}\right) \\
& =\iint_{\mathbb{R}^{2}} \varphi\left(y_{1}, y_{2}\right) \mathbf{1}_{\left[x_{2}, M_{2}\right]}\left(y_{2}\right) \times\left(\int_{\left(M_{1},+\infty[\right.} \mu_{T}\left(d u_{1}-x_{1}, d y_{2}-x_{2}\right)\right) \delta_{0}\left(d y_{1}\right)
\end{aligned}
$$

setting $\left(u_{1}, y_{2}\right)=\left(x_{1}+z_{1}, x_{2}+z_{2}\right)$ for the third line, and using $\varphi\left(0, y_{2}\right)=$ $\int_{\mathbb{R}} \varphi\left(y_{1}, y_{2}\right) \delta_{0}\left(d y_{1}\right)$ and Fubini's theorem for the last line. (Note that the integration on $\left(M_{1},+\infty\right.$ [ is made with respect to $\left.u_{1}\right)$. This provides Eq.(3). Similar computations provide Eq.(2), Eq.(4) and Eq.(5).

Remark 1 In case $X_{t}$ admits a probability density function (p.d.f.) $f_{t}$ with respect to Lebesgue measure, we get:

$$
\mathcal{Q}_{2}(x, d y)=\mathbf{1}_{\left[x_{2}, M_{2}\right]}\left(y_{2}\right) \times\left(\int_{M_{1}}^{+\infty} f_{T}\left(u_{1}-x_{1}, y_{2}-x_{2}\right) d u_{1}\right) \delta_{0}\left(d y_{1}\right) d y_{2},
$$

with similar formulas for the other terms.

In case $X_{t}$ takes range in $\mathbb{N}^{2}$ and $M=\left(M_{1}, M_{2}\right) \in \mathbb{N}^{2}$, we have:

$$
\begin{aligned}
& \mathcal{Q}_{2}(x, d y) \\
& =\sum_{k_{2}=x_{2}}^{M_{2}}\left(\sum_{k_{1}=M_{1}+1}^{+\infty} \mathbb{P}\left(X_{T}^{(1)}=k_{1}-x_{1}, X_{T}^{(2)}=k_{2}-x_{2}\right)\right) \delta_{0}\left(d y_{1}\right) \delta_{k_{2}}\left(d y_{2}\right)
\end{aligned}
$$

for all $x=\left(x_{1}, x_{2}\right) \in \mathbb{N}^{2}$ such that $0 \leq x_{1} \leq M_{1}$ and $0 \leq x_{2} \leq M_{2}$. 
Remark 2 For the two-component system with dependent components, a natural question is: what is the influence of the dependence between the two components on the performance of the maintained system? More specifically, consider two different systems with identical characteristics, except from the fact that the two components are more dependent in one system than in the other (perhaps because of different levels of stress, which induces more or less dependence between components). Then, is it possible to get comparison results between performance indicators of the two maintained systems, such as reliability or cost e.g.? In mathematical terms, assume that $X=\left(X_{t}^{(1)}, X_{t}^{(2)}\right)_{t \geq 0}$ and $\tilde{X}=\left(\tilde{X}_{t}^{(1)}, \tilde{X}_{t}^{(2)}\right)_{t \geq 0}$ are two bivariate subordinators with identically distributed marginal processes $\left(X_{t}^{(i)}\right)$ and $\left(\tilde{X}_{t}^{(i)}\right), i=1,2$, and such that the components of $X=\left(X_{t}^{(1)}, X_{t}^{(2)}\right)_{t \geq 0}$ are "less dependent" than the components of $\tilde{X}=\left(\tilde{X}_{t}^{(1)}, \tilde{X}_{t}^{(2)}\right)_{t>0}$. Following [9], this may be specified through assuming that $X_{t}$ is smaller than $\tilde{X}_{t}$ in the sense of the concordance order, namely that $F_{t} \leq \tilde{F}_{t}$ (or $\bar{F}_{t} \leq \widetilde{\bar{F}}_{t}$, equivalently), where we add $\sim$ to refer to $\tilde{X}$. To get comparison results on reliability/cost indicators of the two maintained systems, a reasonable way might be to get comparison results between processes $Y$ and $\tilde{Y}$ (with clear notations). However, taking $y_{1}=0$ and $0<y_{2} \leq M_{2}$, we have:

$$
\begin{aligned}
F_{Y_{T}}\left(0, y_{2}\right) & =\mathbb{P}\left(M_{1}<X_{T}^{(1)} ; M_{2}<X_{T}^{(2)}\right)+\mathbb{P}\left(M_{1}<X_{T}^{(1)} ; X_{T}^{(2)} \leq y_{2}\right) \\
& =\bar{F}_{T}\left(M_{1}, M_{2}\right)+\bar{F}_{T}^{(1)}\left(M_{1}\right)-\bar{F}_{T}\left(M_{1}, y_{2}\right)
\end{aligned}
$$

and, in general, there is no reason why $F_{Y_{T}}\left(0, y_{2}\right)$ should be comparable with $F_{\tilde{Y}_{t}}\left(0, y_{2}\right)$. As a consequence, assuming that, without maintenance, the components of one system are less dependent than the components of the other one (with the same characteristics otherwise), the respective levels of the two maintained systems are not comparable (at least in a general setting). Hence, we do not expect the respective reliability/cost indicators of the two maintained systems to be comparable either. This is confirmed by numerical experiments from Section 4, where an example is provided, for which the probability of simultaneous replacement (which is envolved in cost) is not monotone with respect to the dependence between components (see Example 3).

\subsection{The cost function on a finite horizon time}

The total cost on a given time interval depends on the number of replacements (simultaneous or not), on the down-time duration and on the number of inspections. The cost is typically computed on some specified time interval of the form $[0, t)$, and in case $t=n T, n \geq 1$, no replacement cost is considered at time $t$. (Indeed, we do not want to replace components once the horizon time $t$ is reached ). Of course, this does not infer on the mean down time, which is the same on $[0, t]$ or $[0, t)$.

For $x=\left(x_{1}, x_{2}\right) \in\left[0, M_{1}\right] \times\left[0, M_{2}\right]$ and given that the system starts from $Y_{0}=x$, we set:

- $C(x,[0, t))=$ mean cumulated cost on $[0, t)$; 
- $U(x,[0, t))=$ mean cumulated down time on $[0, t)(=$ cumulated unavailability on $[0, t))$;

- $R_{12}(x, n T)=$ mean number of simultaneous replacement of both components at time $n T$ (= probability of simultaneous replacement at time $n T$ because one single simultaneous replacement may occur at time $n T$ );

- $R_{i}(x, n T)=$ mean number of replacement of the $i$-th component at time $n T$ (= probability of replacement of the $i$-th component at time $n T$ ).

With these notations, we get the following expression for the cost function.

Proposition 2 Let $x=\left(x_{1}, x_{2}\right) \in\left[0, M_{1}\right] \times\left[0, M_{2}\right]$. Given that the system starts from $Y_{0}=x$, the mean cost on $[0, t)$ is:

$$
\begin{aligned}
C(x,[0, t)) & =c_{u} U(x,[0, t))+\left(c_{1}+c_{r}\right) \sum_{n: n T<t} R_{1}\left(x_{1}, n T\right) \\
& +\left(c_{2}+c_{r}\right) \sum_{n: n T<t} R_{2}\left(x_{2}, n T\right)-c_{r} \sum_{n: n T<t} R_{12}(x, n T)+c_{p}\left\lfloor\frac{t}{T}\right\rfloor,
\end{aligned}
$$

where $\left\lfloor\frac{t}{T}\right\rfloor$ stands for the integer part of $\frac{t}{T}$ (with $\left\lfloor\frac{t}{T}\right\rfloor \leq \frac{t}{T}<\left\lfloor\frac{t}{T}\right\rfloor+1$ ).

Proof. With our notations, the probability that only the first component is replaced at time $n T$ (given that $Y_{0}=x$ ) is:

$$
R_{1}(x, n T)-R_{12}(x, n T) .
$$

We get:

$$
\begin{aligned}
C(x,[0, t)) & =c_{u} U(x,[0, t))+\left(c_{1}+c_{r}\right) \sum_{n: n T<t}\left[R_{1}\left(x_{1}, n T\right)-R_{12}(x, n T)\right] \\
& +\left(c_{2}+c_{r}\right) \sum_{n: n T<t}\left[R_{2}\left(x_{2}, n T\right)-R_{12}(x, n T)\right] \\
& +\left(c_{1}+c_{2}+c_{r}\right) \sum_{n: n T<t} R_{12}(x, n T)+c_{p}\left\lfloor\frac{t}{T}\right\rfloor
\end{aligned}
$$

which may be synthetized into Eq.(6).

We now have to compute the different quantities involved in $C(x,[0, t))$, which is done in the following proposition.

Proposition 3 Let $x=\left(x_{1}, x_{2}\right) \in\left[0, M_{1}\right] \times\left[0, M_{2}\right]$. Given that the system starts from $Y_{0}=x$, for the first period, we have:

$$
\begin{aligned}
R_{i}(x, T) & =\bar{F}_{T}^{(i)}\left(M_{i}-x_{i}\right), \text { for } i=1,2, \\
R_{12}(x, T) & =\bar{F}_{T}\left(M_{1}-x_{1}, M_{2}-x_{2}\right), \\
U_{P}(x,[0, t)) & =\int_{0}^{t} \bar{F}_{u}\left(L_{1}-x_{1}, L_{2}-x_{2}\right) d u \text { for } t \leq T, \\
U_{S}(x,[0, t)) & =t-\int_{0}^{t} F_{u}\left(L_{1}-x_{1}, L_{2}-x_{2}\right) d u \text { for } t \leq T,
\end{aligned}
$$


where subscripts $P$ and $S$ refer to the parallel and series cases, respectively.

For $n \geq 2$, we have the following Markov renewal equations:

$$
R_{i}\left(x_{i}, n T\right)=\int_{\mathbb{R}_{+}} R_{i}\left(y_{i},(n-1) T\right) Q^{(i)}\left(x_{i}, d y_{i}\right)
$$

for $i=1,2$ and $x_{i} \in\left[0, M_{i}\right]$ and

$$
\begin{aligned}
& R_{12}(x, n T)=\iint_{\mathbb{R}_{+}^{2}} R_{12}(y,(n-1) T) \mathcal{Q}(x, d y), \\
& U(x,[T, t))=\iint_{\mathbb{R}_{+}^{2}} U(y,[0, t-T)) \mathcal{Q}(x, d y)
\end{aligned}
$$

for $x=\left(x_{1}, x_{2}\right) \in\left[0, M_{1}\right] \times\left[0, M_{2}\right]$ and $t>T$, where $U=U_{S}$ or $U_{P}$.

Proof. The two first equations are clear, noting that

$$
\begin{aligned}
R_{12}(x, T) & =\mathbb{P}\left(x_{1}+X_{T}^{(1)}>M_{1}, x_{2}+X_{T}^{(2)}>M_{2}\right) \\
& =\bar{F}_{T}\left(M_{1}-x_{1}, M_{2}-x_{2}\right)
\end{aligned}
$$

(with a similar reasoning for the first one). As for the third and fourth equations, for $t \leq T$, we have for the series case (e.g.):

$$
\begin{aligned}
U_{S}(x,[0, t)) & =\mathbb{E}_{x}\left(\int_{0}^{t}\left(1-\mathbf{1}_{\left[0, L_{1}\right] \times\left[0, L_{2}\right]}\left(X_{u}\right)\right) d u\right) \\
& =t-\int_{0}^{t} \mathbb{P}_{x}\left(X_{u}^{(1)} \leq L_{1}, X_{u}^{(2)} \leq L_{2}\right) d u \\
& =t-\int_{0}^{t} F_{u}\left(L_{1}-x_{1}, L_{2}-x_{2}\right) d u .
\end{aligned}
$$

From the second period, conditioning on the whole history at time $T\left(\mathcal{F}_{T}=\right.$ $\left.\sigma\left(Y_{s}, s \leq T\right)\right)$ and using the Markov property at time $T$, we get for all $n \geq 2$ :

$$
\begin{aligned}
R_{12}(x, n T) & =\mathbb{E}_{x}\left[\mathbf{1}_{\left\{Y_{n T}^{(1)}>M_{1}, Y_{n T}^{(2)}>M_{2}\right\}}\right] \\
& =\mathbb{E}_{x}\left[\mathbb{E}_{x}\left(\mathbf{1}_{\left\{Y_{n T}^{(1)}>M_{1}, Y_{n T}^{(2)}>M_{2}\right\}} \mid \mathcal{F}_{T}\right)\right] \\
& =\mathbb{E}_{x}\left[\mathbb{E}_{x}\left(\mathbf{1}_{\left\{Y_{n T}^{(1)}>M_{1}, Y_{n T}^{(2)}>M_{2}\right\}} \mid Y_{T}\right)\right] \\
& =\mathbb{E}_{x}\left[R_{12}\left(Y_{T},(n-1) T\right)\right] \\
& =\iint_{\mathbb{R}_{+}^{2}} R_{12}(y,(n-1) T) \mathcal{Q}(x, d y) .
\end{aligned}
$$

Similar arguments may be used for the other quantities.

Such results allow to recursively compute the different quantities involved in the cost, using discretized versions of the underlying transition kernels and of the different quantities. 


\subsection{The cost on an infinite horizon time}

Setting $c(I)$ to be the random cumulated cost on some time interval $I$, the point here is to prove existence and to compute the asymptotic unitary cost per unit time, which is defined by

$$
C_{\infty}=\lim _{t \rightarrow \infty} \frac{c([0, t))}{t}
$$

As a first step, one can note that each time both components are simultaneously replaced, the system is as good as new, and its future evolution is stochastically identical to that of the initial system and independent of its past. The stochastic process $Y=\left(Y_{t}\right)_{t \geq 0}$ hence appears as a regenerative process, where simultaneous replacements of both components are regeneration times. The length of a generic cycle is $\tau T$, where

$$
\tau=\inf \left(n \geq 1: Y_{n T}=(0,0)\right) .
$$

Lemma 4 Under the assumption $\bar{F}_{T}\left(M_{1}, M_{2}\right)>0$, the mean length of a generic cycle is finite: $\mathbb{E}(\tau)<+\infty$.

Proof. Let

$$
\alpha=\mathbb{P}(\tau>1)=1-\bar{F}_{T}\left(M_{1}, M_{2}\right),
$$

with $\alpha \in[0,1)$ by assumption. For all $n \in \mathbb{N}^{*}$, we have:

$$
\begin{aligned}
\mathbb{P}(\tau>n+1) & =\mathbb{P}(\tau>n ; \tau>n+1) \\
& =\mathbb{E}\left[\mathbf{1}_{\{\tau>n\}} \mathbb{E}\left(\mathbf{1}_{\{\tau>n+1\}} \mid \mathcal{F}_{n T}\right)\right] \\
& =\mathbb{E}\left[\mathbf{1}_{\{\tau>n\}} h\left(Y_{n T}\right)\right]
\end{aligned}
$$

with

$$
\begin{aligned}
h(x) & =\mathbb{E}\left(\mathbf{1}_{\{\tau>n+1\}} \mid Y_{n T}=x\right) \\
& =\mathbb{P}_{x}(\tau>1) \\
& =1-\bar{F}_{T}\left(M_{1}-x_{1}, M_{2}-x_{2}\right) \\
& \leq \alpha
\end{aligned}
$$

due to the Markov property. We hence have

$$
\mathbb{P}(\tau>n+1) \leq \mathbb{E}\left[\mathbf{1}_{\{\tau>n\}} \alpha\right]=\alpha \mathbb{P}(\tau>n)
$$

for all $n \geq 1$ and consequently $\mathbb{P}(\tau>n) \leq \alpha^{n}$, all $n \geq 1$. This provides:

$$
\mathbb{E}(\tau)=\sum_{n=0}^{\infty} \mathbb{P}(\tau>n) \leq \sum_{n=0}^{\infty} \alpha^{n}<\infty .
$$

As $\left(Y_{t}\right)_{t \geq 0}$ is a regenerative process with finite mean length cycle $\mathbb{E}(\tau T)=$ $T \mathbb{E}(\tau)$, we derive from classical renewal theory [10] the almost sure existence of the asymptotic unitary cost $C_{\infty}$ and its following expression:

$$
C_{\infty}=\frac{\mathbb{E}(c([0, \tau T)))}{\mathbb{E}(\tau T)}
$$

The point now is to compute this quantity. With that aim, we follow [11] and we express it with respect to the stationary distribution of the Markov chain $\left(Y_{n T}\right)_{n \geq 0}$. 
Proposition 5 Under the assumption $\bar{F}_{T}\left(M_{1}, M_{2}\right)>0$, the Markov chain $\left(Y_{n T}\right)_{n \geq 0}$ admits a unique stationary distribution $\pi$ (say). Besides:

$$
C_{\infty}=\frac{\mathbb{E}_{\pi}[C(\cdot,[0, T))]}{T}
$$

where

$$
\mathbb{E}_{\pi}[C(\cdot,[0, T))]=\iint_{\left[0, M_{1}\right] \times\left[0, M_{2}\right]} C(x,[0, T)) \pi(d x)
$$

and where we recall that $C(x,[0, T))$ stands for the mean cumulated cost on $[0, T)$, given that the system starts from $x$, see Section 3.2.

Proof. This proof follows step by step [11]. We recall the reasoning here, for sake of completeness.

First, with the help of Lemma 4, we know that the Markov chain almost surely comes back to state $(0,0)$ in a finite time. So that $\left(Y_{n T}\right)_{n \geq 0}$ is a Harris chain. According to [10], there consequently exists a stationary measure $\nu$ for the Markov chain $\left(Y_{n T}\right)_{n \geq 0}$, which is given by

$$
\nu(A)=\mathbb{E}\left(\sum_{n=0}^{\tau-1} \mathbf{1}_{\left\{Y_{n T} \in A\right\}}\right)
$$

for any Borel set $A$ of $\left[0, M_{1}\right] \times\left[0, M_{2}\right]$, where $\nu$ is unique up to a multiplicative constant. As $\nu\left(\left[0, M_{1}\right] \times\left[0, M_{2}\right]\right)=\mathbb{E}(\tau)$ is finite, the measure $\nu$ can be normalized into one single stationary distribution, which is provided by

$$
\pi=\frac{\nu}{\nu\left(\left[0, M_{1}\right] \times\left[0, M_{2}\right]\right)}=\frac{\nu}{\mathbb{E}(\tau)} .
$$

Also

$$
\begin{aligned}
\mathbb{E}(c([0, \tau T))) & =\mathbb{E}\left(\sum_{i=0}^{\tau-1} c([i T,(i+1) T))\right) \\
& =\sum_{i=0}^{+\infty} \mathbb{E}\left(c([i T,(i+1) T)) \mathbf{1}_{\{\tau>i\}}\right)
\end{aligned}
$$

Now, for all $i \in \mathbb{N}$, using that the event $\{\tau>i\}$ belongs to $\mathcal{F}_{i T}$ and due to the Markov property at time $i T$, we get:

$$
\begin{aligned}
& \mathbb{E}\left(c([i T,(i+1) T)) \mathbf{1}_{\{\tau>i\}}\right) \\
& =\mathbb{E}\left(\mathbb{E}\left(c([i T,(i+1) T)) \mid \mathcal{F}_{i T}\right) \mathbf{1}_{\{\tau>i\}}\right) \\
& =\mathbb{E}\left(C\left(Y_{i T},[0, T)\right) \mathbf{1}_{\{\tau>i\}}\right) .
\end{aligned}
$$

This provides

$$
\begin{aligned}
\mathbb{E}(c(\tau T)) & =\mathbb{E}\left(\sum_{i=0}^{\tau-1} C\left(Y_{i T},[0, T)\right)\right) \\
& =\iint_{\left[0, M_{1}\right] \times\left[0, M_{2}\right]} C(x,[0, T)) \nu(d x)
\end{aligned}
$$


due to Eq.(10), from where we derive Eq.(9), using $\nu=\mathbb{E}(\tau) \pi$ (see Eq.(11)).

To compute the asymptotic unitary cost provided by Eq.(9), we now have left to compute the stationary distribution $\pi$ of the Markov chain $\left(Y_{n T}\right)_{n \in \mathbb{N}}$. The results are provided under the assumption that the distribution $\mu_{T}$ of $X_{T}$ admits a density with respect to Lebesgue measure. This assumption is only meant to make the results more readable but is in no case mandatory.

Proposition 6 Assume that the distribution $\mu_{T}$ admits a density $f_{T}$ with respect to Lebesgue measure and let

$$
\begin{aligned}
& g\left(y_{1}, y_{2}\right)=\int_{M_{1}}^{\infty} f_{T}\left(u_{1}-y_{1}, y_{2}\right) d u_{1}, \\
& h\left(y_{1}, y_{2}\right)=\int_{M_{2}}^{\infty} f_{T}\left(y_{1}, u_{2}-y_{2}\right) d u_{2}, \\
& k\left(y_{1}, y_{2}\right)=\bar{F}_{T}\left(M_{1}-y_{1}, M_{2}-y_{2}\right),
\end{aligned}
$$

for all $0 \leq y_{1} \leq M_{1}$ and all $0 \leq y_{2} \leq M_{2}$.

The invariant distribution $\pi$ of the Markov chain $\left(Y_{n T}\right)_{n \geq 0}$ is of the shape

$$
\begin{aligned}
\pi(d x)= & a_{12}\left(x_{1}, x_{2}\right) d x_{1} d x_{2}+a_{1}\left(x_{1}\right) d x_{1} \delta_{0}\left(d x_{2}\right) \\
& +a_{2}\left(x_{2}\right) \delta_{0}\left(d x_{1}\right) d x_{2}+a_{4} \delta_{0}\left(d x_{1}\right) \delta_{0}\left(d x_{2}\right),
\end{aligned}
$$

where $\left(a_{12}, a_{1}, a_{2}, a_{4}\right)$ is solution of the following set of integral equations:

$$
\begin{aligned}
& a_{12}\left(x_{1}, x_{2}\right)=\int_{0}^{x_{1}} \int_{0}^{x_{2}} a_{12}\left(y_{1}, y_{2}\right) f_{T}\left(x_{1}-y_{1}, x_{2}-y_{2}\right) d y_{1} d y_{2} \\
& \quad+\int_{0}^{x_{2}} a_{2}\left(y_{2}\right) f_{T}\left(x_{1}, x_{2}-y_{2}\right) d y_{2}+\int_{0}^{x_{1}} a_{1}\left(y_{1}\right) f_{T}\left(x_{1}-y_{1}, x_{2}\right) d y_{1}+a_{4} f_{T}\left(x_{1}, x_{2}\right), \\
& a_{1}\left(x_{1}\right)=\int_{0}^{x_{1}} \int_{0}^{M_{2}} a_{12}\left(y_{1}, y_{2}\right) h\left(x_{1}-y_{1}, y_{2}\right) d y_{1} d y_{2} \\
& \quad+\int_{0}^{M_{2}} a_{2}\left(y_{2}\right) h\left(x_{1}, y_{2}\right) d y_{2}+\int_{0}^{x_{1}} a_{1}\left(y_{1}\right) h\left(x_{1}-y_{1}, 0\right) d y_{1}+a_{4} h\left(x_{1}, 0\right) \\
& a_{2}\left(x_{2}\right)=\int_{0}^{M_{1}} \int_{0}^{x_{2}} a_{12}\left(y_{1}, y_{2}\right) g\left(y_{1}, x_{2}-y_{2}\right) d y_{1} d y_{2} \\
& \quad+\int_{0}^{x_{2}} a_{2}\left(y_{2}\right) g\left(0, x_{2}-y_{2}\right) d y_{2}+\int_{0}^{M_{1}} a_{1}\left(y_{1}\right) g\left(y_{1}, x_{2}\right) d y_{1}+a_{4} g\left(0, x_{2}\right)
\end{aligned}
$$

and

$$
\begin{aligned}
a_{4}= & \int_{0}^{M_{1}} \int_{0}^{M_{2}} a_{12}\left(y_{1}, y_{2}\right) k\left(y_{1}, y_{2}\right) d y_{1} d y_{2} \\
& +\int_{0}^{M_{2}} a_{2}\left(y_{2}\right) k\left(0, y_{2}\right) d y_{2}+\int_{0}^{M_{1}} a_{1}\left(y_{1}\right) k\left(y_{1}, 0\right) d y_{1}+a_{4} k(0,0)
\end{aligned}
$$

for all $0 \leq x_{1} \leq M_{1}$ and all $0 \leq x_{2} \leq M_{2}$, 
with the additional normalizing constraint:

$$
\int_{0}^{M_{1}} \int_{0}^{M_{2}} a_{12}\left(y_{1}, y_{2}\right) d y_{1} d y_{2}+\int_{0}^{M_{1}} a_{1}\left(y_{1}\right) d y_{1}+\int_{0}^{M_{2}} a_{2}\left(y_{2}\right) d y_{2}+a_{4}=1 .
$$

Proof. Writing $\pi(d x)=(\pi \mathcal{Q})(d x)$, where $\mathcal{Q}$ is provided in Proposition 1, we have

$$
\pi(d x)=\sum_{i=1}^{4} \iint_{\left[0, M_{1}\right] \times\left[0, M_{2}\right]} \pi(d y) \mathcal{Q}_{i}(y, d x)
$$

which may be written as:

$$
\begin{aligned}
\pi(d x)= & \left(\int_{0}^{x_{1}} \int_{0}^{x_{2}} f_{T}\left(x_{1}-y_{1}, x_{2}-y_{2}\right) \pi(d y)\right) d x_{1} d x_{2} \\
& +\left(\int_{0}^{M_{1}} \int_{0}^{x_{2}} g\left(y_{1}, x_{2}-y_{2}\right) \pi(d y)\right) \delta_{0}\left(d x_{1}\right) d x_{2} \\
& +\left(\int_{0}^{x_{1}} \int_{0}^{M_{2}} h\left(x_{1}-y_{1}, y_{2}\right) \pi(d y)\right) d x_{1} \delta_{0}\left(d x_{2}\right) \\
& +\left(\int_{0}^{M_{1}} \int_{0}^{M_{2}} k\left(y_{1}, y_{2}\right) \pi(d y)\right) \delta_{0}\left(d x_{1}\right) \delta_{0}\left(d x_{2}\right) .
\end{aligned}
$$

This proves that the invariant distribution is of the form Eq.(12).

Next, introducing Eq.(12) into Eq.(18) and identifying the terms with respect to $d x_{1} d x_{2}, d x_{1} \delta_{0}\left(d x_{2}\right), \delta_{0}\left(d x_{1}\right) d x_{2}$ and $\delta_{0}\left(d x_{1}\right) \delta_{0}\left(d x_{2}\right)$, we obtain the expressions of $a_{12}, a_{1}, a_{2}$ and $a_{4}$ provided by Eq.(13)-Eq.(16), respectively. Eq.(17) simply is $\int_{0}^{M_{1}} \int_{0}^{M_{2}} \pi(d y)=1$.

The stationary distribution $\pi$ (or rather $\left(a_{12}, a_{1}, a_{2}, a_{4}\right)$ ) now appears as solution of a set of Volterra integral equations of the second kind. Taking $f_{T}$ smooth enough as in the next section, a discretized version of $\pi$ may be computed using an iterative scheme based on the method of successive approximations $[12,13]$, as in [7].

\section{Numerical experiments}

\subsection{Bivariate Gamma process}

The same specific model as in $[6,14]$ is here used for the numerical experiments, which we call bivariate Gamma process. We recall its construction, for sake of completeness. We first remind that a univariate Gamma process with parameters $(a, b)$ (where $a, b>0$ ) is a subordinator $Z$ such that for every $t \geq 0$, the random variable $Z_{t}$ is Gamma distributed $\Gamma(a t, b)$ with p.d.f.:

$$
f_{a t, b}(x)=\frac{1}{\Gamma(a t)} b^{a t} e^{-b x} x^{a t-1} 1_{\{x>0\}} .
$$

We only envision the case $b=1$ in the following, which is no restriction. 
Starting from three independent univariate Gamma processes $\left(Z_{t}^{(i)}\right)_{t \geq 0}$ with parameters $\left(\alpha_{i}, 1\right)$ for $i=1,2,3$ (where $\alpha_{1}, \alpha_{2}, \alpha_{3}>0$ ), we set

$$
\begin{aligned}
& X_{t}^{(1)}=Z_{t}^{(1)}+Z_{t}^{(3)}, \\
& X_{t}^{(2)}=Z_{t}^{(2)}+Z_{t}^{(3)} .
\end{aligned}
$$

The process $\left(X_{t}\right)_{t \geq 0}=\left(X_{t}^{(1)}, X_{t}^{(2)}\right)_{t \geq 0}$ then is a bivariate subordinator with Gamma marginal processes and marginal parameters $\left(a_{i}, 1\right)$ where $a_{i}=\alpha_{i}+\alpha_{3}$ for $i=1,2$. Pearson's correlation coefficient between the two random variables $X_{t}^{(1)}$ and $X_{t}^{(2)}$ is independent of $t$ and given by

$$
\rho=\frac{\alpha_{3}}{\sqrt{a_{1} a_{2}}} .
$$

We consequently have $\alpha_{1}=a_{1}-\rho \sqrt{a_{1} a_{2}}, \alpha_{2}=a_{2}-\rho \sqrt{a_{1} a_{2}}$ and $\alpha_{3}=\rho \sqrt{a_{1} a_{2}}$, with $0 \leq \rho \leq \rho_{\max }=\min \left(\sqrt{\frac{a_{1}}{a_{2}}}, \sqrt{\frac{a_{2}}{a_{1}}}\right)$. Two alternate parameterizations hence are available for $\left(X_{t}\right)_{t \geq 0}$ : either $\left(\alpha_{1}, \alpha_{2}, \alpha_{3}\right)$ or $\left(a_{1}, a_{2}, \rho\right)$. Besides, all the dependence between the marginal processes is contained in the linear correlation coefficient $\rho$.

\subsection{Validation of the results}

We here present a few numerical experiments to validate our theoretical results and their practical implementation, especially for the invariant distribution $\pi$, which is the most technical to compute.

In all the section, the parameters of the bivariate Gamma process are $\left(a_{1}, a_{2}, \rho\right)=$ $(4,4,0.6)$. The preventive maintenance thresholds are $M_{1}=0.5$ and $M_{2}=0.3$ and the inspection period is $T=0.6$. (No other parameters needed here).

As a first case, the probabilities of replacement of one or two components are computed at first and second inspection times, via the results of Proposition 3 with discretization steps $h_{1}=h_{2}=0.01$. The results are displayed in Table 1, as well as those obtained by Monte-Carlo (MC) simulation, with $10^{5}$ histories and 95\% confidence intervals (CI). All results are coherent. Note that, though both components share a common deterioration parameter $\left(a_{1}=a_{2}\right)$, their preventive thresholds are different $\left(M_{1}>M_{2}\right)$, which leads to higher replacement probabilities for the second component. As expected, the probability of simultaneous replacements $\left(R_{12}\right)$ is lower than the probability of replacing at least one component $\left(R_{i}, i=1,2\right)$.

In Table 2 , results are displayed for the asymptotic probabilities of replacement of one or two components by an inspection, namely for $\mathbb{E}_{\pi}\left(R_{i}(\cdot, T)\right)$, $i=1,2$ and $\mathbb{E}_{\pi}\left(R_{12}(\cdot, T)\right)$, with

$$
\mathbb{E}_{\pi}\left(R_{12}(\cdot, T)\right)=\int_{0}^{M_{1}} \int_{0}^{M_{2}} R_{12}(x, T) \pi(d x)
$$

and similar expressions for the other terms. The distribution $\pi$ has been numerically computed as explained at the end of Section 3.3 and $R_{12}(x, T)$ is computed via Proposition 3, just as for Table 1. Results are also provided by MC simulations, with $10^{7}$ histories. For these MC results, we have used the fact 
that $\pi$ is the asymptotic distribution of the Markov chain $\left(Y_{n T}\right)_{n \in \mathbb{N}}$ and waited until the chain is stabilized:

$$
\mathbb{E}_{\pi}\left(R_{12}(\cdot, T)\right)=\lim _{n \rightarrow+\infty} \mathbb{E}\left(R_{12}\left(Y_{n T}, T\right)\right) \simeq \mathbb{E}\left(R_{12}\left(Y_{N T}, T\right)\right)
$$

for large $N$. Here again, all results are coherent.

Finally, the convergence of the distribution of $\left(Y_{n T}\right)_{n>0}$ towards $\pi$ is illustrated in Figure 2 through the numerical convergence of the mean rate of simultaneous replacements on $[0, n T]$ per unit time towards the asymptotic rate:

$$
\lim _{n \rightarrow+\infty} \frac{1}{n T} \sum_{i=1}^{n} R_{12}(0, i T)=\frac{1}{T} \mathbb{E}_{\pi}\left(R_{12}(\cdot, T)\right) .
$$

To obtain this figure, all computations have been performed using the theoretical results of the previous sections (no Monte-Carlo simulation).

Based on the numerical results of this section (and on others not displayed here), one may think that the practical implementation of our theoretical results is correct.

\subsection{Examples}

Parameters for all examples are displayed in Table 3, as well as the system structure when necessary (for computations of mean down times and costs).

Example 1 Two parts of the invariant distribution $\pi$ of $\left(Y_{n T}\right)_{n>0}$ are displayed in Figure 3: functions $a_{1}\left(x_{1}\right)$ and $a_{12}(x)$, which are the p.d.f.' $s$ of $\pi$ with respect to $d x_{1} \delta_{0}\left(d x_{2}\right)$ (replacement of component 2 only) and of $d x_{1} d x_{2}$ (no replacement), respectively. With the chosen parameters, we observe that $a_{1}\left(x_{1}\right)$ is increasing with $x_{1}$ and that $a_{12}(x)$ is concave. Also, we get $a_{4} \simeq 0.89$, so that the probability of simultaneous replacements of both components is here quite high for large times.

This example illustrates the variations of the mean rate of simultaneous replacements per unit time with respect to the period $T$, both in the asymptotic and finite time cases (see Figure 4). For the finite horizon case $\left[0, t_{0}\right)$ (with $t_{0}=4$ fixed), this mean rate of replacements is not continuous with respect to $T$ (Figure 4a). The discontinuity points are the points $T$ such that there exists some number $n$ satisfying $t_{0}=n T$, because at these points, we do not consider possible replacements at $t_{0}$. As for the asymptotic case, the rate of simultaneous replacements seems continuous with respect to $T$ (Figure $4 \mathrm{~b}$ ).

Figure 5a next shows that, as expected, the unitary cumulated mean down time on $\left[0, t_{0}\right)$, namely $U\left(0,\left[0, t_{0}\right)\right) / t_{0}$, is increasing with $T$. Though we have not studied it from a theoretical point of view, one might indeed think that $U\left(0,\left[0, t_{0}\right)\right)$ (with $t_{0}$ fixed) increases with $T$. Figure 5 b shows that the asymptotic unavailability $\mathbb{E}_{\pi}(U(\cdot,[0, T])) / T$ is also increasing with $T$.

The asymptotic unitary cost is plotted in Figure $6 b$ as a function of $T$. The function is convex and admits a single minimum at $T_{o p t}^{\infty} \simeq 0.52$. On the finite horizon $\left[0, t_{0}\right)$, the mean unitary cost as a function of $T$ is not continuous (Figure $6 a$ ) and the minimum is at $T_{\text {opt }} \simeq 0.03$. The optimal inspection periods hence are very different for the infinite and finite horizons. 
Example 2 The influence of the preventive maintenance thresholds $\left(M_{1}, M_{2}\right)$ on the cost function is here studied, in the case of a series system. We take $c_{p}=c_{1}=0$ and consider several cases for $\left(c_{2}, c_{u}, c_{r}\right)$. Figures have similar shapes for the finite and infinite horizons. To save some space, they are only provided for the infinite case. As we can see, the cost function may be convex (Figure 7), concave (Figure 8) or more complicated (Figure 9). So, it seems hard to say anything about the shape of the cost function with respect to the preventive maintenance thresholds $\left(M_{1}, M_{2}\right)$ in a general setting. This may be due to the fact that the mean number of replacements and the down-time durations may have reverse concavity with respect to $\left(M_{1}, M_{2}\right)$.

Example 3 We finally look at the influence of the dependence between the two components on the probability of simultaneous replacements of both components. Let us recall that, for the bivariate Gamma model, this dependence is measured by Pearson's correlation $\rho$, see Eq.(19). The probability of simultaneous replacements of both components is plotted with respect to $\rho$ in Figure 10. We can see that it is not monotone with respect to $\rho$, which is in concordance with Remark 2.

\section{Conclusion}

We here considered a two-component system, with deterioration modelled by a bivariate subordinator. A condition-based preventive replacement policy has been proposed, which is rather classical in the univariate setting, with deterioration modelled by a Gamma process. In the bivariate case, it leads to imperfect repairs at the system level, which highly complicates the study. As an example, the four parts of the stationary distribution of the underlying Markov chain have been characterized as the single solution of a set of four Volterra integral equations of the second kind, and the numerical computation of the stationary distribution is much more demanding than in the univariate setting. In the same way, the variations of the cost functions with respect to each parameter have been observed to be complicated and sometimes not easily predictable (see Figures 9 or 10 e.g.). The qualitative results obtained in a univariate setting cannot hence be easily adapted to the bivariate case and there consequently is a clear need for further development.

\section{Acknowledgements}

Hai Ha PHAM thanks the Conseil Régional d'Aquitaine (France) which supports her research work. This work was also supported for both authors by the French National Research Agency (ANR), AMMSI project, ref. ANR 2011 BS01-021. The authors thank both referees and the Editor-in-Chief for their constructive remarks which improved the presentation of the paper.

\section{References}

[1] M. Abdel-Hameed (1975). A Gamma wear process. IEEE Transactions on Reliability 24(2): 152-153. 
[2] N. Singpurwalla (1995). Survival in dynamic environments. Statistical Science 10(1): 86-103.

[3] J. M. Van Noortwijk (2009). A survey of the application of Gamma processes in maintenance. Reliability Engineering $\mathcal{E}$ System Safety 94(1): $2-21$.

[4] F. A. Buijs, J. W. Hall, J. M. Van Noortwijk, P. B. Sayers (2005). Time dependent reliability analysis of flood defences using Gamma processes. In Augusti, G., Schüeller, G. I., \& Ciampoli, M., editors, Safety and Reliability of Engineering Systems and Structures; Proceedings of ICOSSAR 9: 22092216, Rotterdam, Millpress.

[5] N. Ebrahimi (2004). Indirect assessment of the bivariate survival function. Annals of the Institute of Statistical Mathematics 56(3): 435-448.

[6] S. Mercier, H. H. Pham (2012). A preventive maintenance policy for a continuously monitored system with correlated wear indicators. European Journal of Operational Research 222(2): 263-272.

[7] B. Castanier, A. Grall, C. Bérenguer (2005). A condition-based maintenance policy with non-periodic inspections for a two-unit series system. Reliability Engineering \&3 System Safety 87(1): 109-120.

[8] J. Bertoin (1996). Lévy processes. Cambridge Tracts in Mathematics 121, Cambridge University Press, Cambridge.

[9] Nicole Bäuerle, Anja Blatter, Alfred Müller (2008) Dependence properties and comparison results for Lévy processes, Mathematical Methods of Operations Research 67: 161-186.

[10] S. Asmussen (2003). Applied probability and queues. Applications of Mathematics 51. Springer-Verlag, New York, second edition.

[11] C. Cocozza-Thivent (2000). Convergence de fonctionnelles de processus semi-régénératifs, Prépublications de l'Université de Marne la Vallée 02/2000 (in French).

[12] R. Kress (1999). Linear integral equations. Applied Mathematical Sciences 82, Springer Verlag.

[13] T.O. Shaposhnikova, R.S. Anderssen, S.G. Mikhlin (1958). Integral equation - a reference context. Noordhoff International Publishing Leyden.

[14] S. Mercier, C. Meier-Hirmer, M. Roussignol (2012). Bivariate Gamma wear processes for track geometry modelling, with application to intervention scheduling. Structure and Infrastructure Engineering 8(4): 357-366. 


\section{TABLES \& FIGURES}

\begin{tabular}{|c|c|c|c|}
\hline & $\begin{array}{c}\text { Formula } \\
h_{1}=0.01, h_{2}=0.01\end{array}$ & $\begin{array}{l}\text { MC simulation } \\
\quad N=10^{5}\end{array}$ & MC 95\% CI \\
\hline$R_{1}(0, T)$ & 0.9551 & 0.9555 & {$\left[\begin{array}{ll}0.9542 & 0.9568\end{array}\right]$} \\
\hline$R_{1}(0,2 T)$ & 0.9569 & 0.9572 & {$\left[\begin{array}{lll}0.9562 & 0.9587\end{array}\right]$} \\
\hline$R_{2}(0, T)$ & 0.9849 & 0.9850 & {$\left[\begin{array}{lll}0.9842 & 0.9857\end{array}\right]$} \\
\hline$R_{2}(0,2 T)$ & 0.9851 & 0.9848 & {$\left[\begin{array}{lll}0.9840 & 0.9856\end{array}\right]$} \\
\hline$R_{12}(0, T)$ & 0.9456 & 0.9456 & {$\left[\begin{array}{lll}0.9441 & 0.9470\end{array}\right]$} \\
\hline$R_{12}(0,2 T)$ & 0.9465 & 0.9469 & {$\left[\begin{array}{lll}0.9455 & 0.9483\end{array}\right]$} \\
\hline
\end{tabular}

Table 1: Probabilities of replacement at first and second inspection times

\begin{tabular}{|cccc|}
\hline & $\begin{array}{c}\text { Formula } \\
h_{1}=0.01, h_{2}=0.01\end{array}$ & $\begin{array}{c}\text { MC Simulation } \\
N=10^{7}\end{array}$ & MC 95\% CI \\
\hline $\mathbb{E}_{\pi}\left(R_{1}(., T)\right)$ & 0.9568 & 0.9568 & {$[0.95550 .9569]$} \\
\hline $\mathbb{E}_{\pi}\left(R_{2}(., T)\right)$ & 0.9851 & 0.9851 & {$[0.98500 .9851]$} \\
\hline $\mathbb{E}_{\pi}\left(R_{12}(., T)\right)$ & 0.9467 & 0.9466 & {$[0.94640 .9467]$} \\
\hline
\end{tabular}

Table 2: Asymptotic probabilities of replacement at inspection times

\begin{tabular}{lccccccccccccccc}
\hline & Structure & $a_{1}$ & $a_{2}$ & $\rho$ & $L_{1}$ & $L_{2}$ & $M_{1}$ & $M_{2}$ & $T$ & $c_{1}$ & $c_{2}$ & $c_{r}$ & $c_{p}$ & $c_{u}$ & $t_{0}$ \\
\hline Ex.1 & - & 4 & 9 & 0.5 & - & - & 0.6 & 0.9 & 0.5 & - & - & - & - & - & - \\
Ex. 1 & Parallel & 4 & 4 & 0.5 & 0.7 & 0.6 & 0.5 & 0.5 & - & 1 & 1 & 1 & 0.01 & 10 & 4 \\
Ex. 2 & Series & 4 & 9 & 0.5 & 1.2 & 1.4 & - & - & 0.5 & 0 & - & - & 0 & - & $4 \mathrm{~T}$ \\
Ex. 3 & - & 4 & 9 & - & - & - & 4 & 5 & 1 & - & - & - & - & - & $4 \mathrm{~T}$ \\
\hline
\end{tabular}

Table 3: Parameter values used in the examples 


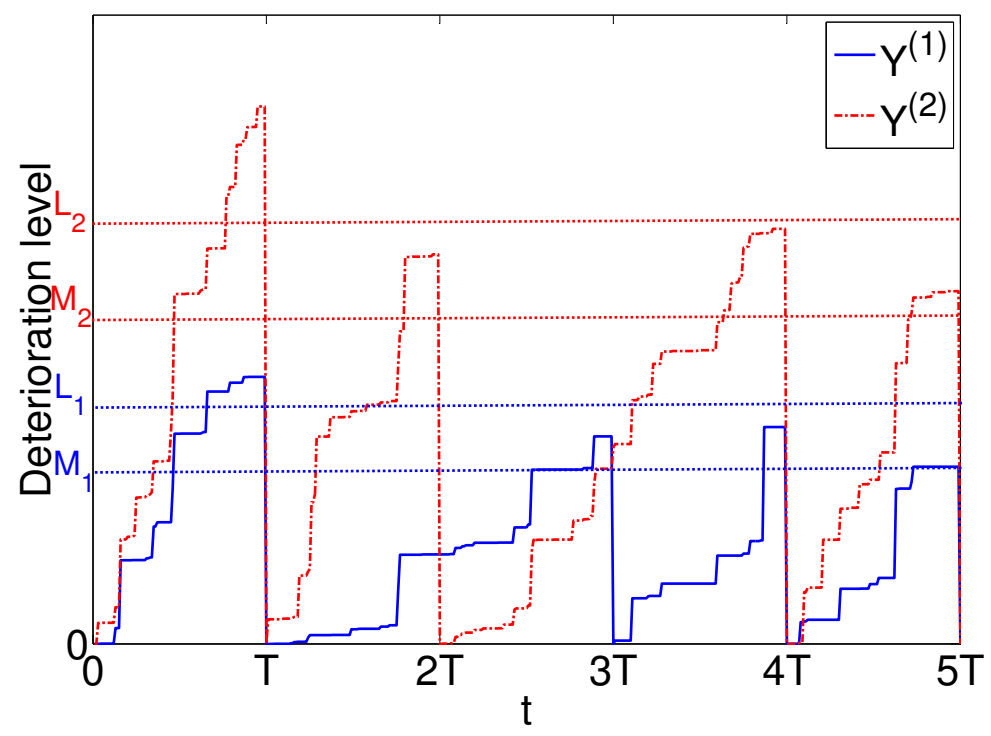

Figure 1: The preventive maintenance policy

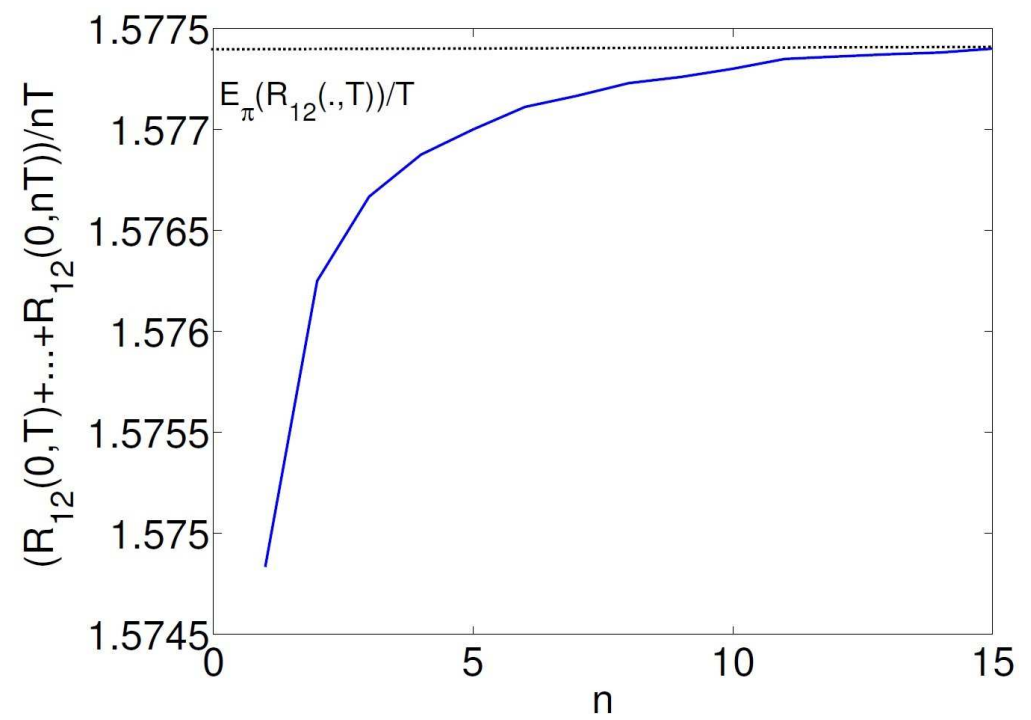

Figure 2: Illustration of the convergence of the Markov chain $\left(Y_{n T}\right)_{n \geq 1}$ 

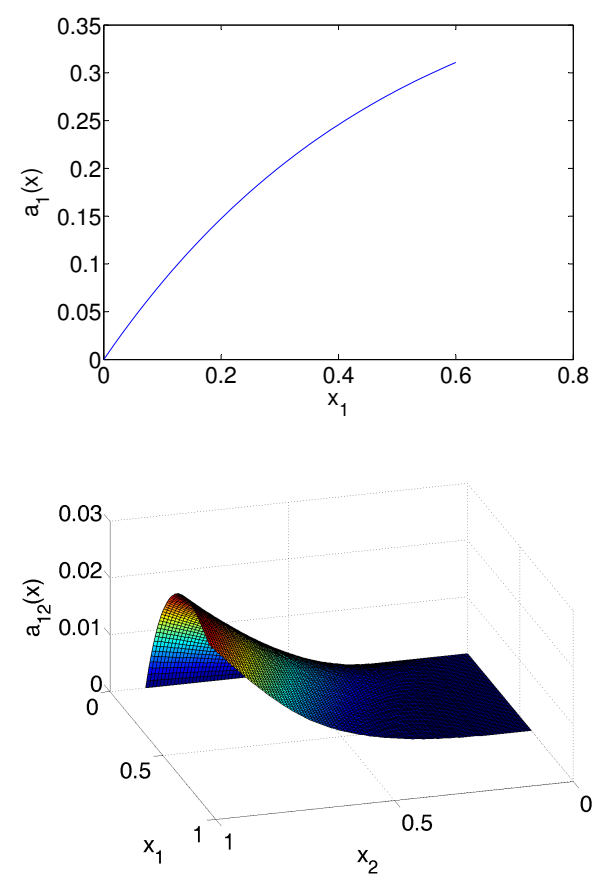

Figure 3: Parts of the invariant distribution of $\left(Y_{n T}\right)_{n \geq 0}$, Example 1

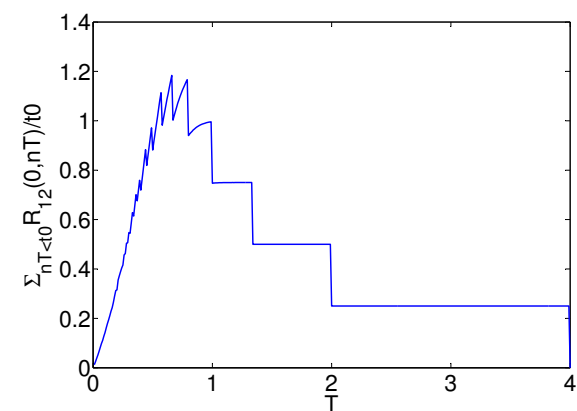

(a) Finite time case

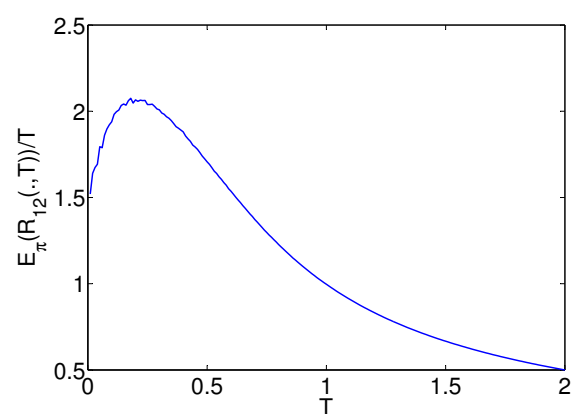

(b) Asymptotic case

Figure 4: Mean rate of simultaneous replacements as a function of $T$, Example 


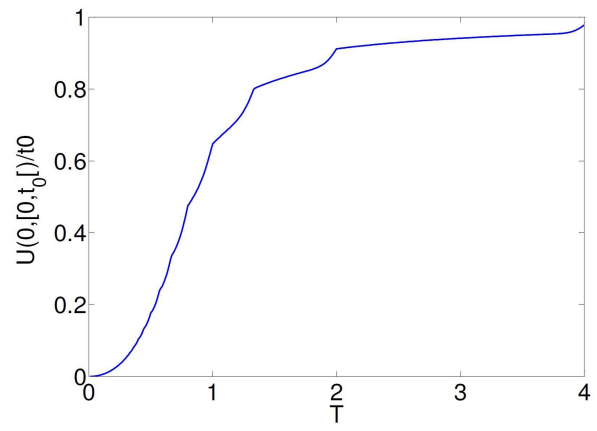

(a) Finite time case

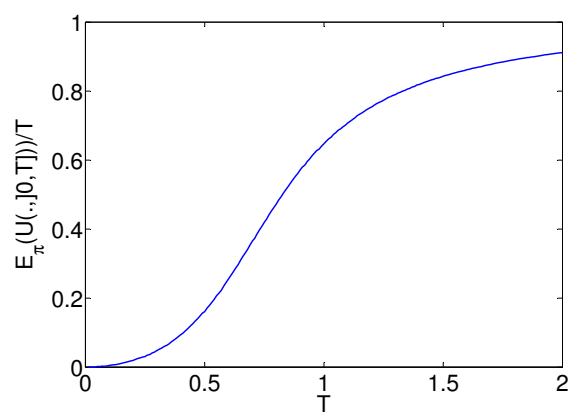

(b) Asymptotic case

Figure 5: Unitary mean down time per unit time as a function of $T$, Example 1

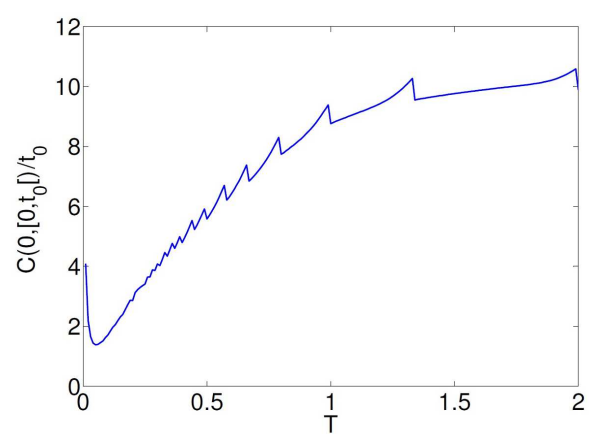

$\begin{array}{ll}\text { (a) Finite time case } & \text { (b) Asymptotic case }\end{array}$

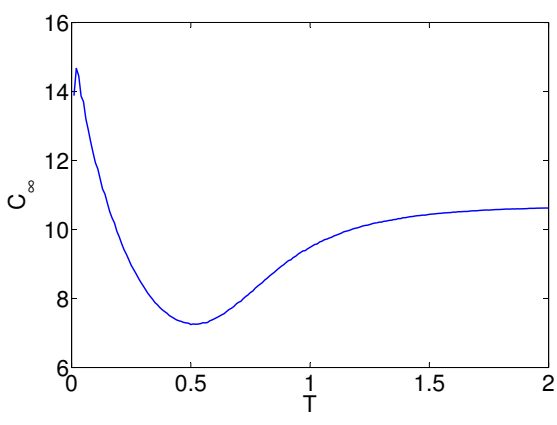

(b) Asymptotic case

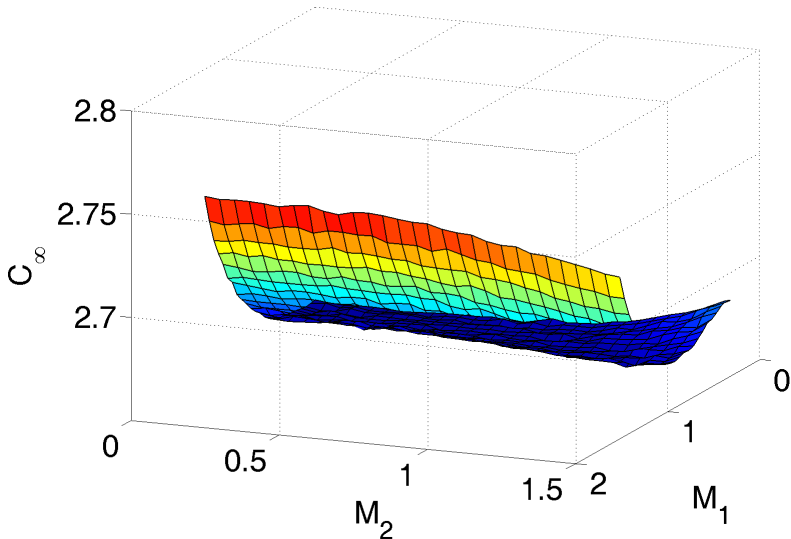

Figure 7: The asymptotic cost rate with respect to $\left(M_{1}, M_{2}\right)$, Example 2, $c_{2}=0$, $c_{r}=1, c_{u}=2$ 


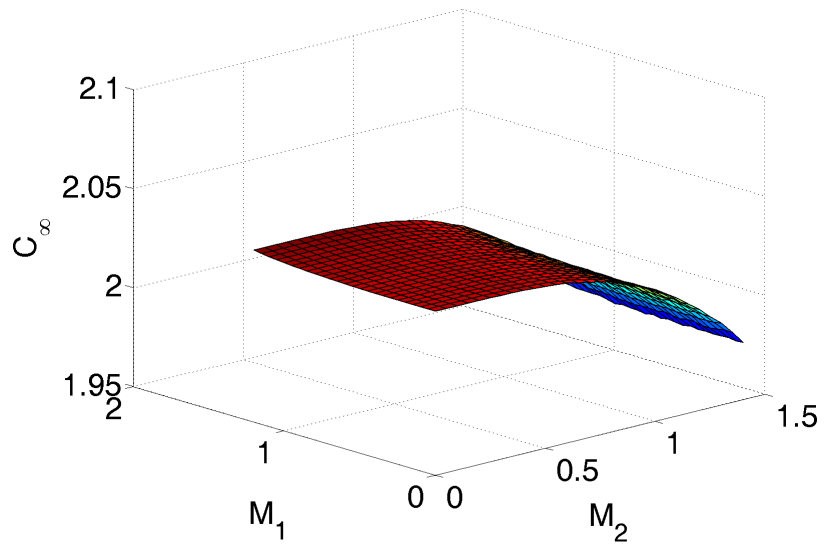

Figure 8: The asymptotic cost rate with respect to $\left(M_{1}, M_{2}\right)$, Example $2, c_{2}=1$, $c_{r}=0, c_{u}=0.1$

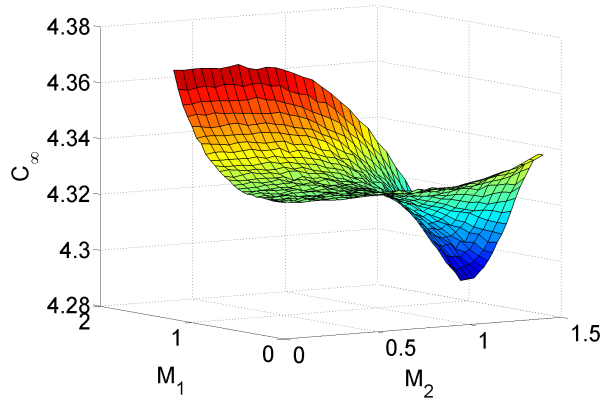

Figure 9: The asymptotic cost rate with respect to $\left(M_{1}, M_{2}\right)$, Example $2, c_{2}=0$, $c_{r}=2, c_{u}=1$ 


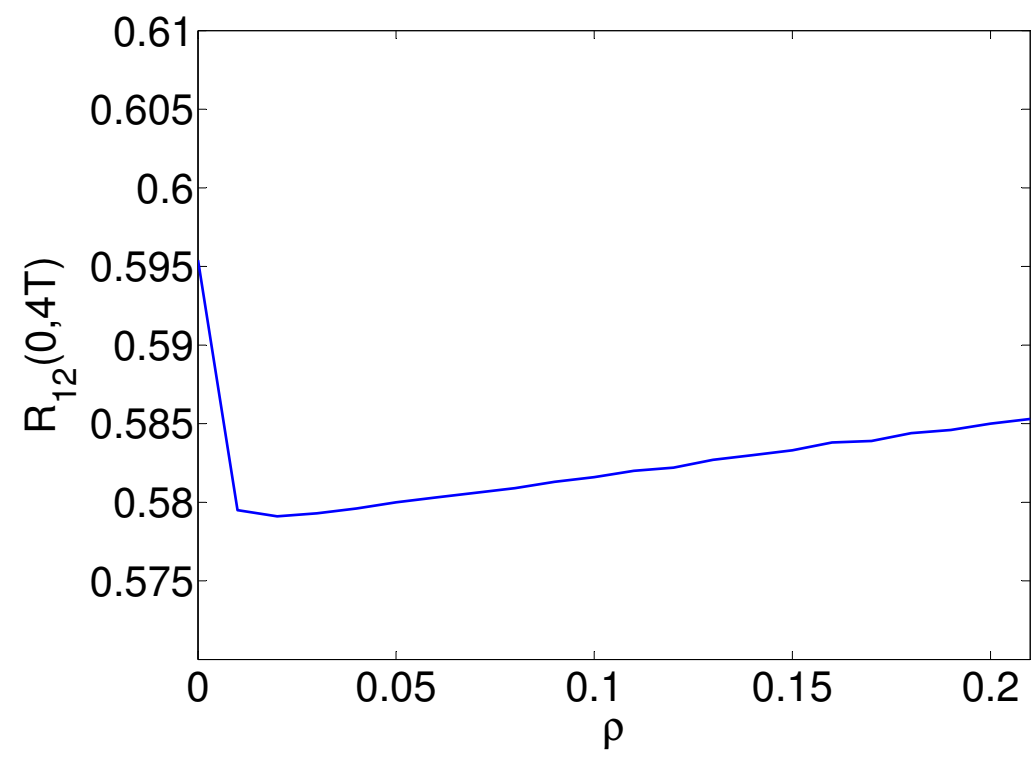

Figure 10: Probability of simultaneous replacement of both components with respect to $\rho$, Example 3 